\title{
Virtual Reality for AAL Services Interaction Design and Evaluation
}

\author{
Pilar Sala ${ }^{1}$, Felix Kamieth ${ }^{2}$, Juan Bautista Mocholí ${ }^{1}$, and Juan Carlos Naranjo ${ }^{1}$ \\ ${ }^{1}$ Instituto de Aplicaciones de las Tecnologías de la Información y de las Comunicaciones \\ Avanzadas. ITACA Technological Centre, \\ Universidad Politécnica de Valencia, Edificio 8G \\ Camino de Vera s/n 46022 Valencia, Spain \\ \{msalaso, juamocag, jcnaranjo\} @itaca.upv.es \\ ${ }^{2}$ Fraunhofer Institute for Computer Graphics Research IGD, Fraunhoferstraße 5, \\ 64283 Darmstadt, Germany \\ felix.kamiethaigd.fraunhofer.de
}

\begin{abstract}
AAL Solutions are not part of mainstream industry yet, being one of the main reasons the complexity of the technologies involved in relation to its targeted beneficiaries, the elderly, and its acceptance by them. Applying HCD methodologies for user involvement and creating physical prototypes is both costly and time consuming, particularly in this domain that combines software artifacts, devices and physical environments. VR techniques are very suited to create Virtual Prototypes that offer the beneficiaries the possibility to visualize and interact with proposed solutions before they exist. This paper presents the results of VAALID project in developing this approach of creating tools for design and simulation of AAL Solutions using VR and Mixed Reality, supporting the early involvement of beneficiaries in the process.
\end{abstract}

Keywords: AAL systems, Virtual Reality, Mixed Reality, simulation, user involvement.

\section{Introduction}

Ambient Assisted Living (AAL) solutions are technological solutions to support elderly and people with disabilities in their everyday lives allowing them to live selfsufficiently at home for a longer period of time.

Currently, the level of deployment of these solutions in real life is still very low, as well as their acceptability by elderly, being one of its main barriers detected the complexity of the technology involved. [1]

In order to overcome this barrier, an integrated user centered design vision is needed. HCD process requires the direct involvement of future beneficiaries from the initial concept through systems design and integration to the prototypes and business models and a continuous revision and adaptation of proposed solution designs.

In AAL domain, where software artifacts, all kind of devices and physical environments are interacting with the beneficiaries, the creation of prototypes is both 
costly and time-consuming, which restricts the development of high-quality solutions that require several iterations through the HCD process.

As an alternative for physical prototyping and testing, Virtual Reality can offer to the beneficiaries the capability to explore and interact in a realistic manner with AAL Solutions before they exist in reality [2].

The VAALID project [3] is a Specific Targeted Research Project supported by the European Commission with the goal of creating an integrated development and simulation environment (VAALID IDE), which can speed up the iterations through the Human Centre Design cycle. The central idea is to create an AAL solution virtually first and then refine this virtual solution with beneficiaries and other stakeholders before turning the prototypes physical.

This paper presents the results of the implementation of such VAALID IDE, focusing on the developed tools for VR representation and simulation of AAL Solutions.

\section{Proposed Approach: VAALID IDE}

VAALID IDE offers an advanced, integrated, computer aided design environment, consisting of the following, components

- An Authoring Tool (AE), that allows designers to create and deploy the components that constitute the interaction structure of an AAL solution. It enables a good overview of the services offered and the ability of fast editing and adaptation to changes, which will occur as the solution goes through the iterations of the HCD process. It is composed by:

- A virtual reality scene supported with virtual devices and sensors, simulating the physical build-up of the environment.

- A modeling framework [4] to describe the active parts available within the simulated solution. It supports models of users, environments, interactions and services. These models provide the functional basis for the simulation of the environment.

- A Virtual Reality Simulation Environment (SE) in which users can navigate through and interact with the simulated scene to test the developed services in its virtual stage. At this point, the designer can already incorporate his target users into the process by letting them experience his solution in the simulation and give valuable feedback for further improvement.

On top of the virtual representation, the simulation environment is also capable of incorporating real devices into the simulation, which improves the range of services that can be tested and gives the developer the opportunity of gradually moving from the virtual to the real implementation of his solution and conduct user tests along the way.

The major innovation of VAALID IDE consists in the special combination and integration of the Authoring Environment and the Simulation Environment, which makes it possible to progress through a virtuous cycle of design-deployment-testing, that provides a tremendous support in the conception, tuning and implementation of AAL solutions. 
In fact, the system allows the dynamic change and fine-tuning of different interaction models within the Authoring Environment and their immediate deployment and testing in the Simulation Environment, thus implementing a rapid application development paradigm within the AAL design process and favoring the application of the HCD approach.

In this way, VAALID IDE will help to bridge the gap between the planning phase of AAL solutions and their testing and evaluation in reality (e.g. in a living lab), bringing in several important advantages:

- Possibility of early, "in silico" detection of potentially costly flaws, before resources are actually committed to implementation and production

- Faster decision making, due to the reduction of the time spent in the designdeployment-testing loop

- Improving the research of innovative solutions, through the conduction of efficient "what-if" analysis

- Potential to address the needs of a vast number of senior citizens' categories, by fast switching among different beneficiaries' interaction profiles at design time

- Management of new types of information not achievable with traditional means, as for instance information on beneficiaries' reactions

These advantages foster a significant advancement of the AAL design process, resulting in higher product quality and innovation, reduced time-to-market and decreased implementation costs, in a relatively young industry, the take up of which is crucially dependent on the improvement of the cost/benefit ratio.

\section{Implementation of Authoring Environment}

The AE groups the development tools that allow the designer to define and describe the individual elements that intervene in the AAL solution. Some of them have been already the subjects of publications $[4,5]$ so we are going to focus on describing the Environment Model builder that is directly related with the VR capabilities of VAALID IDE.

This tool provides the designer with the capability of defining the environment in which the AAL solution will take place includes both physical space and objects placed there.

In order to model the physical space, this editing tool imports the 3D scene from a previously created VRML file, and allows the designer to identify the elements that are relevant for the interaction with the AAL service and assign them the desired properties and behaviour.

Devices are modeled from three different perspectives:

- Semantic: That is the properties the device has (type, location, reference to visual and behaviour representation, functions...)

- Visual: That is the 3D representation of the device. In the VRML it's also defined the interactions it can handle in Virtual Reality simulation by means of animation scripts that are parts of the simulated scene, which can be triggered by external programs and effect changes within the scene, like displaying certain media, opening a door or closing the shades of a window for example 
- Behaviour: That is the workflow associated to the device. It represents the dynamic it offers to the elderly in the AAL solution, defining the possible states of the object, the inputs that will make it change it state and the outputs it will provide to the system [6].

In next figure, two screenshots of AE Environment Model builder are shown; on the left it is the device editor with the semantic properties and the visual representation, while on the right it is the workflow editor to define the behavior.
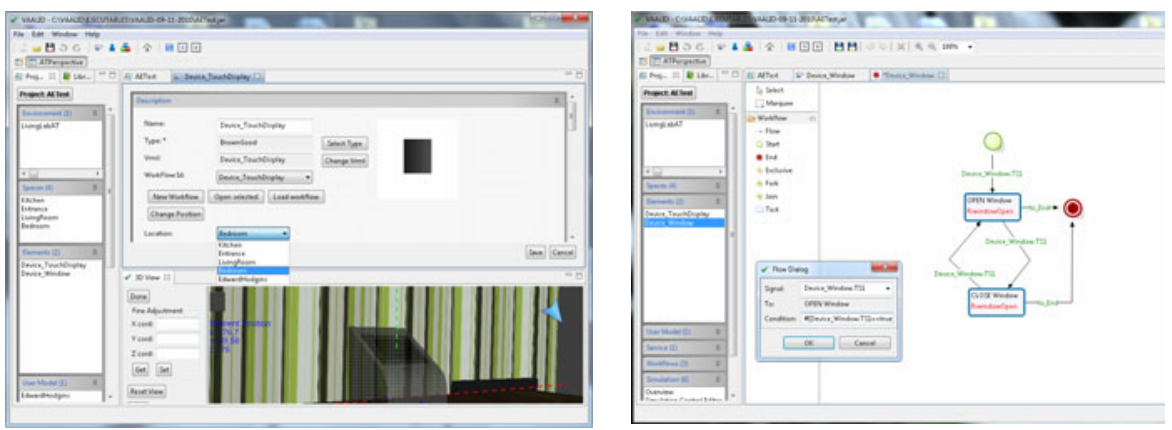

Fig. 1. VAALID Authoring Environment - Device editor (left) and workflow editor (right)

\section{Implementation of Simulation Environment}

The VAALID Simulation Environment serves the simulation of AAL services provided by intelligent environments. This requires the simulation of the physical environment as well as the functional simulation of the devices contained therein and finally the AAL services using the devices available in the environment to provide the environment's system intelligence.

Displaying the 3D-model is only a part of the simulation environment. Figure 2 gives an overview of the different components and their connections. The simulation environment is composed of five main elements:

- The InstantReality Player, which displays the 3D-environment including all simulated devices and sensors and receives end-user inputs through simulation controls.[7]

- The Workflow Engine, which simulates the developed AAL services and the functionalities of the simulated devices and sensors as workflows (similar to statemachines moving from one state to another through a set of predefined available transitions)

- The Process Choreographer, which is the core of the simulation as it connects the Workflow Engine with the simulated equipment in the 3D-scene and the real equipment in the Living Lab [6].

- The Logger, which receives data from the Process Choreographer about the actions of the simulated services and equipment and from the Instant Player about end-user input (like movement through the scene and interaction with the active 
components of the simulated environment) and saves all these results in a file for later analysis.

- The Simulation Control Panel, which allows the user to set the parameters of the simulation environment and view the workflow states and the Logger output while the simulation is running. The user can also manually change the workflow states and thus interact with the running simulation.

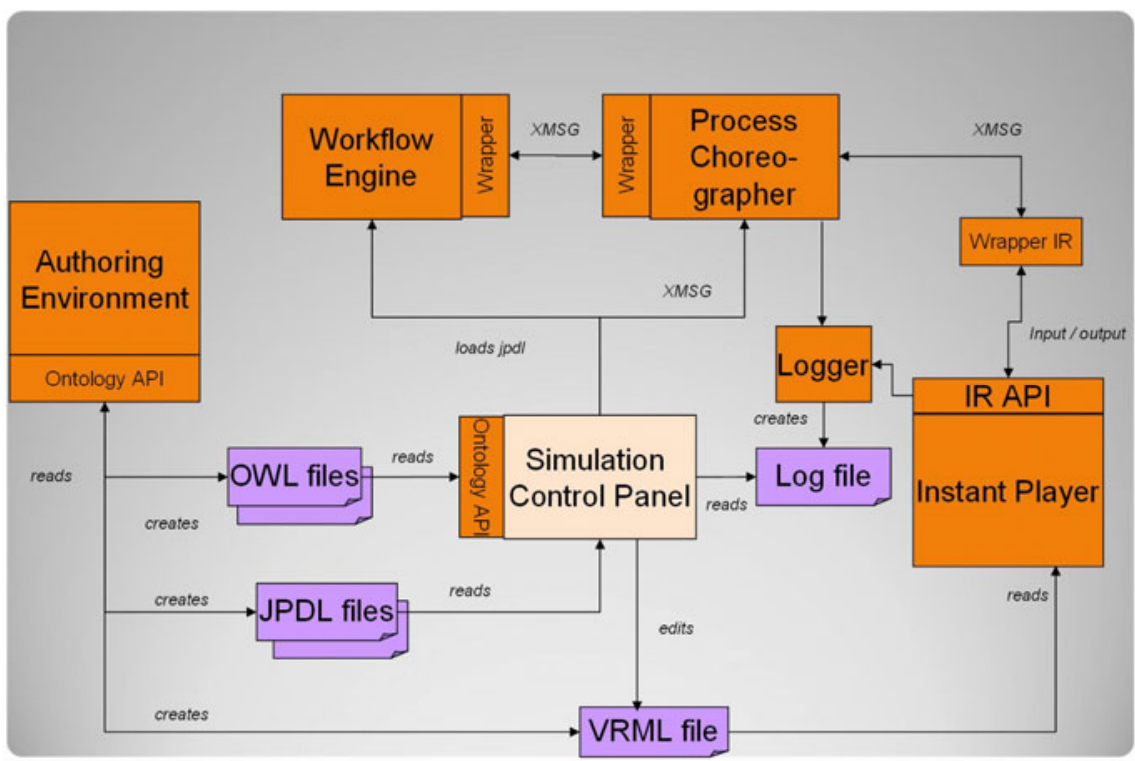

Fig. 2. VAALID Simulation Environment functional architecture

\subsection{Connection between 3D-Simulation and Workflow Engine}

The 3D-Environment running in the InstantReality Player is connected to the workflows running on the workflow engine. The InstantReality Player provides an interface for external programs to exchange events with the running simulation through a network connection. This connection allows the retrieval of events from user inputs to the simulation.

All 3D-models of devices and sensors are created according to a set of specific templates, which allows external applications access to all the active components within a simulated environment. For easy access to the simulation environment an API was developed which connects to the existing interface of the InstantReality Player but allows easy access to the simulated devices and sensors' reactions to user inputs and simulated functionalities.

Figure 3 shows how the connection between devices and sensors in the simulation and external programs is done in detail. Sensors and devices running in the InstantReality Player send data through the API to an external program. To support a flow of information in the other side, too, the external program can then call the devices' services (like opening a door, turning on a light, showing a certain message on a display, etc.). 


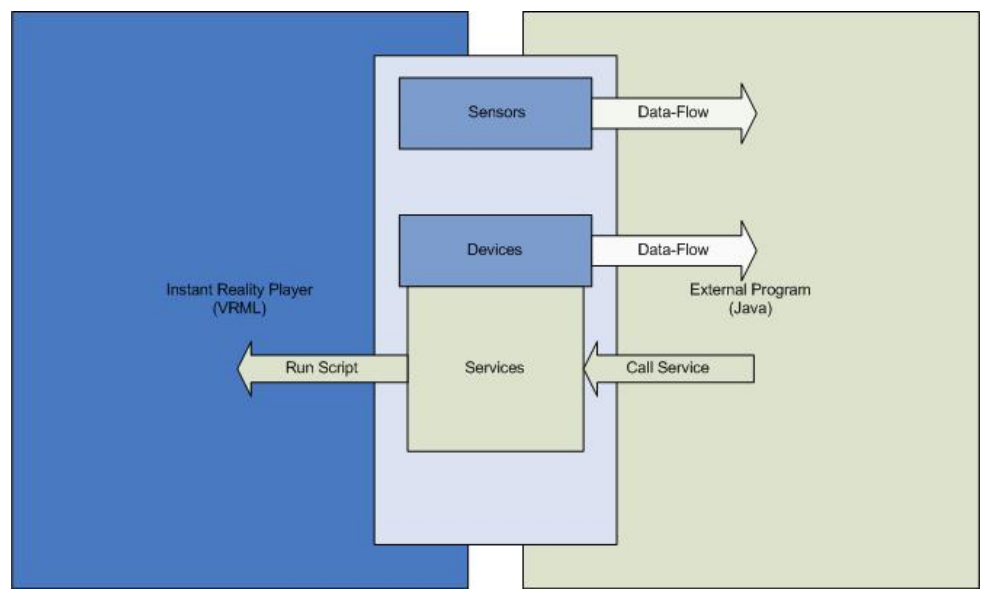

Fig. 3. Interaction between external programs and simulated equipment

\subsection{Simulation Controls Support}

The 3D-simulation serves the purpose of giving an end-user of an intelligent environment a realistic impression of the developed solution to receive feedback on the ongoing development in a fast and cost-effective manner. To give the user a realistic impression of the environment and provide useful feedback to the solution designers, the end-user testing the solution in the simulation environment needs to be able to interact with the solution in a simple and understandable fashion. Since most AAL systems are built with elderly people in mind as end-users the interaction with the simulation environment faces a big challenge in terms of interaction. The user interaction with an intelligent environment is a challenge of its own. On top of this, the simulation environment itself places another barrier between the user and the solution, which makes it necessary to develop a solution that takes different interaction modalities into account and supports a variety of different interaction devices. This ensures the adaptability of the simulation system to further developments in the user interaction field and lets the designer pick the interaction modality most suitable for the evaluation of a specific AAL solution.

To support different interaction devices, a layer was set in between the device and the simulation mapping the inputs from the device to the simulation environment. The interactions with the simulation take place in two ways. The first is the navigation through the environment, which allows forward and backward movement and rotation at different speeds. The second is interaction with devices and sensors in the environment. For this, a virtual hand in the environment is being controlled, which allows the user to open doors, press buttons etc. in the environment.

To ensure adaptability to different interaction modalities, this interaction has been tested so far with different devices both for interaction and navigation. Devices include a gamepad, a special wheelchair, a dance mat and a mobile phone and a space mouse for navigation as well as a Novint Falcon and a gamepad for interaction.

The designer can choose among the different devices supported so far in the Simulation Control Panel, which lets the user configure the simulation before it is started. 
Initial conditions for devices can be set. During a simulation run, the user can take screenshots of the 3D-simulation, interact with the simulation by changing the states of the simulated devices and track the simulation run, logging all user interaction and device and sensor activations.

\subsection{Connection to the Living Lab Verifier}

For improved realism and support of devices and sensors, which cannot be simulated for user interaction testing like on-body sensors, the simulation system also supports the connection to real world devices. In the Simulation Control Panel, the user can choose if the simulation run should contain a real device or its simulated counterpart. This allows the gradual movement from a pure VR simulation to a mixed reality simulation and a complete test of the AAL services in a real living lab with all sensors and devices being real.

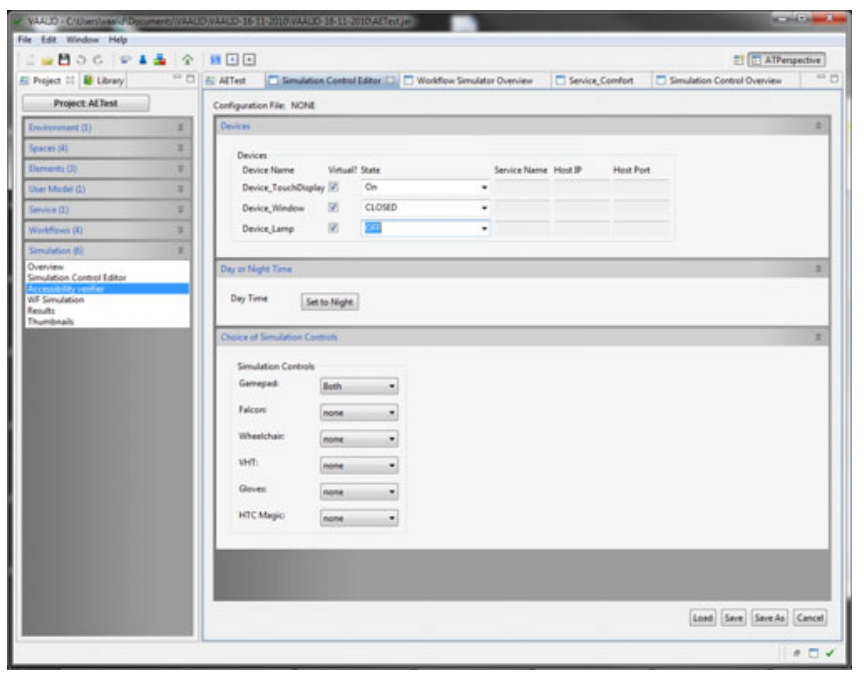

Fig. 4. VAALID Simulation Environment control panel

Real and simulated devices share the connection to the workflow engine running the AAL service. A layer between devices and workflow engine allows a standard interface for in- and outputs from devices to the system intelligence, which simplifies the switch from a virtual to a real device and thus further increases the speed of solution development.

\subsection{Running the Simulation}

The work done by the designer in the Authoring Environment is saved in three types of files: the first set of files are workflow-description files (JPDL-files), which contain the descriptions of the AAL-services as well as the descriptions of the functionalities of the different kinds of devices; the second set of files are ontologies (OWL-files), which contain a semantic description of the environment, its devices and the user 
model; and the third type is a VRML-file which contains the 3D-representation of the environment including all scripts and simulation control connections.

Before the simulation can be started based on this input from the Authoring Environment, the user configures the settings for the simulation using the Simulation Control Panel (SCP). When the user chooses the simulation controls to be used to interact with the simulation, the SCP edits the VRML-file accordingly by adding the code necessary for connecting these input devices with the components of the 3D-model. Also, changes in the device initial state result in editing the VRML-file accordingly.

When the user starts the simulation, the Simulation Control Panel loads the JPDLfiles into the Workflow Engine, the VRML-file into the Instant Player and starts the Process Choreographer and the logger. The Process Choreographer exchanges XMLmessages with the workflow engine and the Instant Player, thus mediating between AAL-services and simulated equipment. During the simulation, the logger receives input from the Process Choreographer and the Instant Player, collecting data on all user inputs through the simulation controls and the system's reactions to them, resulting in a complete log of the entire simulation procedure.

During the simulation, the user can view the workflows simulating environment, equipment and AAL-services in action and see the system reactions to user input live in a graphical display. For this display, the SCP loads the graphical representations from the JPDL-files created by the Authoring Environment. The user can also send messages within the simulation while it is running using this graphical display, which results in sending corresponding messages from the Simulation Control Panel to the Process Choreographer.
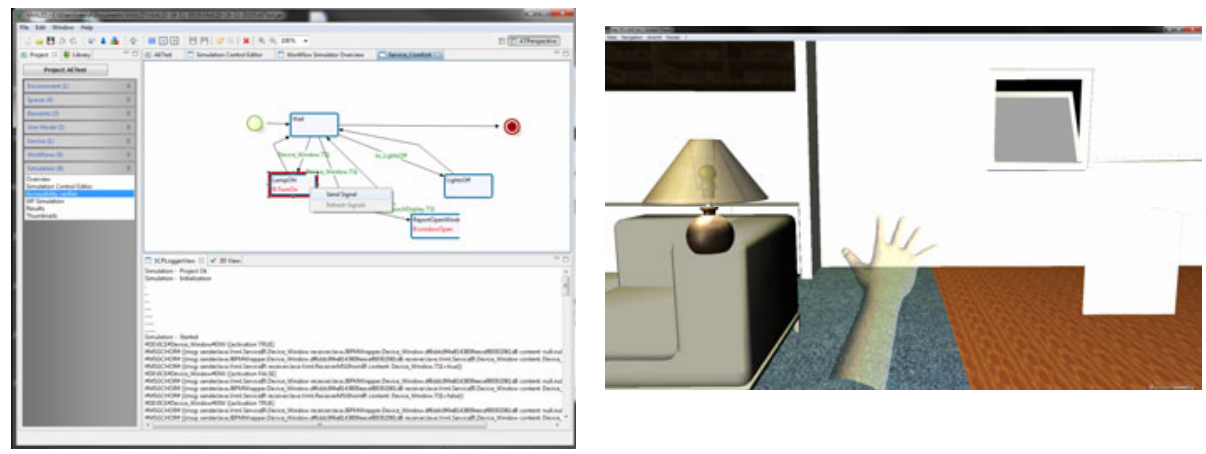

Fig. 5. VAALID SE - workflow simulator (left) and InstantReality Player (right)

After the simulation is complete, the Simulation Control Panel reads the log-file and analyses the simulation results, giving concise feedback to the user based on this analysis.

\section{Conclusions}

In this paper, the results of VAALID project related to the development of a Simulation Environment for AAL Solutions design and evaluation using Virtual and Mixed Reality have been presented. 
The simulation environment is adapted to fit the needs of the most likely test subjects: elderly and people with disabilities, for whom AAL solutions are being developed. They find themselves in an immersive environment equipped with intuitive interaction devices and can thus provide valuable feedback on the developed solutions without being distracted by the simulation environment itself.

Building a virtual Ambient Assisted environment starts with the existing virtual reality model of the physical environment to be simulated. It is equipped with representations of sensors and devices like a real environment is currently set up for AALsystem testing. The simulation of the intelligent services is done using AAL workflows and ontologies, which are modeled graphically and thus allow both for fast setup and editing of solutions.

The VAALID IDE supports the necessary transition from virtual representation to real environment. It allows the creation of a deep sense of presence within the simulation, which is needed for a realistic evaluation of the proposed solution. Connected to this visual system are interaction devices tailored to the use of elderly and persons with disabilities allowing even stronger immersion in the simulation and making the 3D-simulation accessible to a wide variety of test subjects. This is necessary so that the solution is being properly evaluated without much interference from the simulation itself.

Thus VAALID aids in the challenge to develop meaningful AAL solutions, which are available for a wide variety of subjects, making the design process faster and more efficient.

In the time of writing this paper (January 2011) the developed VAALID IDE prototypes were undergoing user evaluation from different perspectives:

- AAL designers and developers were involved to evaluate the user experience of the Authoring Environment,

- Human Factors experts together with elderly were involved to evaluate the user experience of the Simulation Environment

- Key stakeholder of AAL domain were involved to evaluate the impact of such tools in the overall process of designing and building AAL solutions

Only preliminary results are available; the validation phase will conclude in April 2011 and some results will be given in the presentation of the paper in July 2011. The preliminary results give us a great support to continue this research.

Acknowledgments. This work has been partially funded by the European Union in the context of the VAALID project. Our gratitude to the VAALID consortium composed of the following partners: Fh-IGD, UniPR, VOLTA, UPM, UID, SPIRIT and ITACA.

\section{References}

1. Vastenburg, M.H., Visser, T., Vermaas, M., Keyson, D.V.: Designing acceptable assisted living services for elderly users. In: Aarts, E., Crowley, J.L., de Ruyter, B., Gerhäuser, H., Pflaum, A., Schmidt, J., Wichert, R. (eds.) AmI 2008. LNCS, vol. 5355, pp. 1-12. Springer, Heidelberg (2008) 
2. Park, H., Son, J.-S., Lee, K.-H.: Design evaluation of digital consumer products using virtual reality-based functional behavior simulation. Journal of Engineering Design 19(4), 359-375

3. VAALID project (IST-224309), http: / /www. vaalid-project.org/

4. Naranjo, J.-C., Fernandez, C., Sala, P., Hellenschmidt, M., Mercalli, F.: A modelling framework for ambient assisted living validation. In: Stephanidis, C. (ed.) UAHCI 2009. LNCS, vol. 5615, pp. 228-237. Springer, Heidelberg (2009)

5. Mocholí, J.-B., et al.: Ontology for Modeling Interaction in Ambient Assisted Living Environments. In: IFMBE Proceedings, vol. 29, Part 4, pp. 655-658 (2010)

6. Fernández-Llatas, C., et al.: Process choreography for Interaction simulation in Ambient Assisted Living environments. In: IFMBE Proceedings, vol. 29, Part 4, pp. 757-760 (2010)

7. InstantReality Player by Fraunhofer IGD, http: / / www . instantreality . org/ 